\title{
Finite Volume Simulation of the Viscoelastic Fluids At An Expansion Flow
}

\author{
Ameen Ibrahim Galeel ${ }^{\dagger}$, Khudheyer S. Mushatet ${ }^{\ddagger}$ and Hussam Ali Khalif ${ }^{\dagger}$. \\ ${ }^{\dagger}$ Mechanical engineering, Thi-Qar university, Said Dakhil- Nasiriyah -Thi-Qar-Iraq. \\ \$ Mechanical engineering, Thi-Qar university, Bathah- Nasiriyah -Thi-Qar-Iraq.
}

\begin{abstract}
Numerical simulation of viscoelastic fluid flow of the upper-convected Maxwell (UCM) type by finite volume on collected grid arrangement for the steady laminar flow through the 1:4 planer expansion has been obtain for a range of Deborah numbers. The conservation equations and the constitutive equations have been solved by using the finite volume numerical method on a collected grid arrangement with using the power low scheme for the momentum equations and the upwind scheme for the constitutive equations. The solution of the non-linear algebraic equation from the discretization process was obtained by using the Tri-Diagonal Matrix Algorithm (TDMA). The solution was verified with grid refinement. It is discovered that increasing the elasticity level lead to increasing the pressure, stresses and decreases the recirculation zones, where increasing Deborah number from 0 to 1.5 causes the pressure to increase by $2.6 \%$. The finite volume method (FVM) shows that it capable for the numerical simulation of viscoelastic fluid flow with high speed to get the final solution and low computational cost.
\end{abstract}

Keywords: UCM constitutive equation; Expansion flows; Finite-volume method; Viscoelasticity; Collected grid.

\begin{tabular}{|c|c|c|}
\hline & & \\
\hline Symbol & Meaning & Units \\
\hline $\mathrm{H}$ & $\begin{array}{l}\text { Half height of the } \\
\text { channel }\end{array}$ & $\mathrm{m}$ \\
\hline $\mathrm{u}$ & $\mathrm{X}$-axis velocity & $\mathrm{m} / \mathrm{s}$ \\
\hline $\mathrm{V}$ & Y-axis velocity & $\mathrm{m} / \mathrm{s}$ \\
\hline $\mathrm{P}$ & pressure & $\mathrm{N} / \mathrm{m} 2$ \\
\hline De & Deborah number & $\begin{array}{ll}---- \\
\end{array}$ \\
\hline $\operatorname{Re}$ & Reynolds number & ---- \\
\hline
\end{tabular}

\begin{tabular}{|c|c|}
\hline TDMA & Chilcott and Rallison \\
\hline CFD & $\begin{array}{c}\text { Tri-Diagonal Matrix } \\
\text { Algorithm }\end{array}$ \\
\hline E & $\begin{array}{c}\text { Computational fluid } \\
\text { dynamics }\end{array}$ \\
\hline W & East \\
\hline S & West \\
\hline N & South \\
\hline
\end{tabular}

Greek symbols:

\begin{tabular}{|c|c|c|}
\hline Symbol & Meaning & Units \\
\hline$\rho$ & density & $\mathrm{Kg} / \mathrm{m} 3$ \\
\hline$\tau_{x x}$ & X-axis stress & $\mathrm{N} / \mathrm{m} 2$ \\
\hline$\tau_{y y}$ & Y-axis stress & $\mathrm{N} / \mathrm{m} 2$ \\
\hline$\tau_{x y}$ & Shear stress & $\mathrm{N} / \mathrm{m} 2$ \\
\hline$\lambda$ & Relaxation time & ----- \\
\hline$\phi$ & General variable & ----- \\
\hline$\Gamma$ & $\begin{array}{c}\text { Diffusion } \\
\text { coefficient }\end{array}$ & ---- \\
\hline$\nabla$ & Divergence signs & ---- \\
\hline
\end{tabular}

Abbreviation:

\begin{tabular}{|c|c|}
\hline Abbreviation & Meaning \\
\hline FVM & Finite volume method \\
\hline FEM & Finite element method \\
\hline CV & Control volume \\
\hline UCM & Upper convicted Maxwell \\
\hline FENE-P & $\begin{array}{c}\text { Finitely Extensible } \\
\text { Nonlinear Elastic in the } \\
\text { Peterlin approximation }\end{array}$ \\
\hline FENE-CR & Finitely Extensible \\
& Nonlinear Elastic by \\
\hline
\end{tabular}

\section{1- Introduction:}

The simulation of viscoelastic fluid flows is an important field and it is a field of computational fluid dynamic (CFD). The simulation of viscoelastic fluid as well as CFD aims to find the fluid flow variables at different condition such as the pressure field, the stresses and the velocity. This kind of simulations have an important impact and consideration in polymers manufacturing (the polymer's considered as viscoelastic fluid), where the simulations gives an important information about the flow variables. However, in this paper a results are obtained for the simulation of the UCM viscoelastic fluid model by using the finite volume method. The simulation has been done in a 4:1 expansion duct in 2D flow for the creeping flow $(\operatorname{Re}=0.1)$, the simulation have been obtain to Deborah number (De) up to 1.5 .

In the literature a verity of simulations have been obtain for different viscoelastic models. An effort was done by using the finite difference method (FDM) (Gasti, 1975). (Chang, 1979) Also they used the FEM to get the results for their model. A curt result also has been obtained by using spectral finite element methods (S-FEM) (Beris, 1987). In that respect, it was inevitable that the finite volume method (FVM) would also be tried within the 
viscoelastic context, since this method is well-known and has been widely used with success In other fields of computational fluid mechanics. The simulation by using FVM is now widely used in computational fluid dynamics and for the simulation of viscoelastic fluids flow because its simplicity in writing the code for the simulation and it gives results in small time compared to FEM (Oliveira, 2003).

There are a wide range of research on the simulation of viscoelastic fluid flow by using FVM like (Missirlis, 1998) whose his simulation done for the UCM model on collected grid arrangement of a 1:4 sudden planar expansion in the range of De number $(0,1.2$ and 3$)$. Also for the UCM model (Whiteman, 1992) who used the staggered grid arrangement, his simulation done for the range of Weissenberg numbers $(0,1,2.4)$ also in the staggered grid (FU Chun-quan, 2009) he have been use the same model for the simulation of the viscoelastic flow in an abrupt expansion ,his simulation done for the range of Weissenberg numbers $(0,0.6,1.2,3.2)$ for other viscoelastic model. Oliveira, 2003 have been used the modified FENECR model for the simulation of planer expansion with 1:3 expansion ratio his work done for a wide range of $\mathrm{Re}$ number (0.1 to 100) and Weissenberg number up to 2 . Another model used by (Kerim Yapicia, 2010) is the Oldroyd-B viscoelastic fluid flow model his simulation was steady laminar flow in a lid-driven square cavity for a wide range of Reynolds.

In this work the simulation is done for the UCM viscoelastic fluid model by using FVM on collected grid arrangement through 1:4 planer expansions and steady two-dimensional flow. Thus the problem of interest for the 1:4 planer expansion is shown in fig. 1 and the simulation is for the creeping flow, where $(\operatorname{Re}=0.1)$.

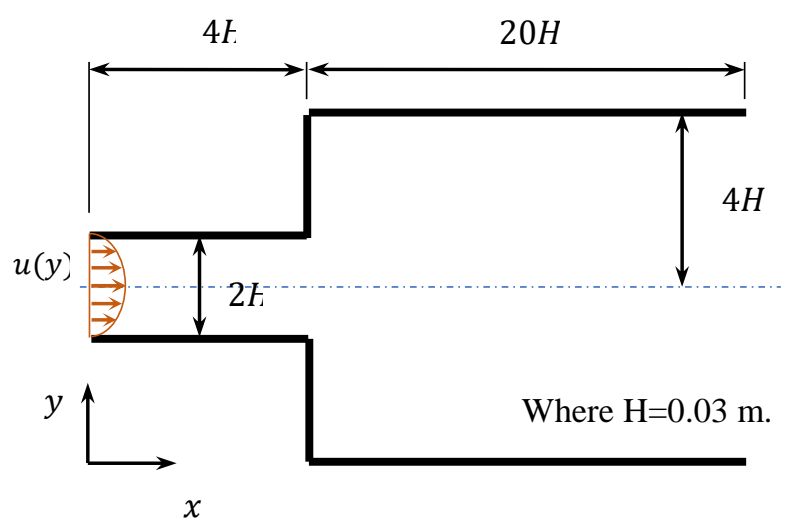

Fig.1 The planer expansion.

\section{Mathematical modeling}

The basic equation that governing the flow of the UCM fluid flow in Cartesian, two-dimensional, laminar and steady flow form are:

The continuity equation [2]:

$$
\begin{gathered}
\frac{\partial u}{\partial x}+\frac{\partial v}{\partial y}=0 \\
\frac{\partial}{\partial x}(\rho u u)+\frac{\partial}{\partial y}(\rho v u)=-\frac{\partial P}{\partial x}+\frac{\partial \tau_{x x}}{\partial x}+\frac{\partial \tau_{x y}}{\partial y} \\
\frac{\partial}{\partial x}(\rho u v)+\frac{\partial}{\partial y}(\rho v v)=-\frac{\partial P}{\partial y}+\frac{\partial \tau_{y y}}{\partial y}+\frac{\partial \tau_{x y}}{\partial x}
\end{gathered}
$$

And the constitutive equations [8]

$$
\begin{aligned}
& \frac{\partial}{\partial x}\left(\lambda u \tau_{x x}\right)+\frac{\partial}{\partial y}\left(\lambda v \tau_{x x}\right)=2 \mu \frac{\partial u}{\partial x}-\left(1-2 \lambda \frac{\partial u}{\partial x}\right) \tau_{x x}+ \\
& 2 \lambda \frac{\partial u}{\partial y} \tau_{x y} \\
& \frac{\partial}{\partial x}\left(\lambda u \tau_{y y}\right)+\frac{\partial}{\partial y}\left(\lambda v \tau_{y y}\right)=2 \mu \frac{\partial v}{\partial y}-\left(1-2 \lambda \frac{\partial v}{\partial y}\right) \tau_{y y}+ \\
& 2 \lambda \frac{\partial v}{\partial x} \tau_{x y} \\
& \frac{\partial}{\partial x}\left(\lambda u \tau_{x y}\right)+\frac{\partial}{\partial y}\left(\lambda v \tau_{x y}\right)=2 \mu\left(\frac{\partial v}{\partial x}+\frac{\partial u}{\partial y}\right)-\tau_{x y}+ \\
& \lambda \frac{\partial v}{\partial x} \tau_{x x}+\lambda \frac{\partial u}{\partial y} \tau_{y y}
\end{aligned}
$$

In these equations, $\mathrm{u}$ is the $\mathrm{x}$-component velocity vector, $\mathrm{v}$ is the $y$-component velocity vector, $P$ is the pressure, $\tau$ is the stress, $\mu$ is the dynamic viscosity of the fluid, $\lambda$ is the relaxation time and $\rho$ is the density.

The dimensionless parameters used in this simulation for the viscoelastic fluid are:

Deborah number (De) [8]

$$
D e=\frac{\lambda U}{H}
$$

And Reynolds number (Re) [2]:

$$
R e=\frac{\rho U H}{\mu}
$$

Where $\mathrm{U}$ is the velocity and $\mathrm{H}$ is the length.

\section{The numerical solution}

By using the FVM The constitutive Eq. (3) and the conservation Eqs. (1) and (2) will be solved together .In finite volume method which is explained in [13], the domain of solution first is divided to small element called finite volume and the conservation equations are integrated over this element. By using gausses theorem to transfer the volume integration of the gradient to a surface integration, where the diffusion and convective terms are used as the coefficient of the algebraic equations and any other terms treated as a source term. Those the algebraic equation arranged in the form [10]:

$$
a_{P} \phi_{P}=\sum_{F} a_{F} \phi_{F}+S_{\phi}
$$


To solve any conservation equation by using the finite volume method first the equations are written in the general form:

$$
\nabla \cdot(\operatorname{mu} \phi)=\nabla \cdot(\Gamma \nabla \phi)+\mathrm{S}
$$

Where $m$ can be the density $\rho$ or relaxation time $\lambda$,it depend on the type of the conservation equation, $\phi$ is the primitive variable , $S$ is the source term and $\Gamma$ is the diffusion coefficient.

\subsection{Discretization of momentum equations}

The momentum equations (1) and (2) before the discretization they will written in the general form by adding a diffusion term for both the sides of the momentum equations [11]:

$$
\begin{array}{r}
\frac{\partial}{\partial x}(\rho u u)+\frac{\partial}{\partial y}(\rho v u)=\frac{\partial}{\partial x}\left(\mu \frac{\partial u}{\partial x}\right)+\frac{\partial}{\partial y}\left(\mu \frac{\partial u}{\partial y}\right)-\frac{\partial P}{\partial x}+ \\
\frac{\partial \tau_{x x}}{\partial x}+\frac{\partial \tau_{x y}}{\partial y}-\frac{\partial}{\partial x}\left(\mu \frac{\partial u}{\partial x}\right)-\frac{\partial}{\partial y}\left(\mu \frac{\partial u}{\partial y}\right) \\
\frac{\partial}{\partial x}(\rho u v)+\frac{\partial}{\partial y}(\rho v v)=\frac{\partial}{\partial y}\left(\mu \frac{\partial v}{\partial y}\right)+\frac{\partial}{\partial x}\left(\mu \frac{\partial v}{\partial x}\right)-\frac{\partial P}{\partial y}+ \\
\frac{\partial \tau_{y y}}{\partial y}+\frac{\partial \tau_{x y}}{\partial x}-\frac{\partial}{\partial y}\left(\mu \frac{\partial v}{\partial y}\right)-\frac{\partial}{\partial x}\left(\mu \frac{\partial v}{\partial x}\right)
\end{array}
$$

The diffusion term was added for the two sides of these equations because there is no diffusion in the momentum equation, where diffusion is an important term to get the stability of finite-volume schemes when integrate the momentum equations [11].

By arrangement the momentum equations in the form of the general conservation equation:

$$
\begin{gathered}
\frac{\partial}{\partial x}(\rho u u)+\frac{\partial}{\partial y}(\rho v u)=\frac{\partial}{\partial x}\left(\mu \frac{\partial u}{\partial x}\right)+\frac{\partial}{\partial y}\left(\mu \frac{\partial u}{\partial y}\right)+S_{x} \\
\frac{\partial}{\partial x}(\rho u v)+\frac{\partial}{\partial y}(\rho v v)=\frac{\partial}{\partial y}\left(\mu \frac{\partial v}{\partial y}\right)+\frac{\partial}{\partial x}\left(\mu \frac{\partial v}{\partial x}\right)+S_{y}
\end{gathered}
$$

Here ;

$$
\begin{gathered}
S_{x}=S_{x 1}+S_{x 2}+S_{x 3}+S_{x 4} \\
S_{y}=S_{y 1}+S_{y 2}+S_{y 3}+S_{y 4}
\end{gathered}
$$

Table.1. The source term parts

\begin{tabular}{|c|c|c|r|}
\hline Term & value & term & value \\
\hline $\mathrm{S}_{\mathrm{x} 1}$ & $-\frac{\partial \mathrm{P}}{\partial \mathrm{x}}$ & $\mathrm{S}_{\mathrm{y} 1}$ & $-\frac{\partial \mathrm{P}}{\partial \mathrm{y}}$ \\
\hline $\mathrm{S}_{\mathrm{x} 2}$ & $\frac{\partial \tau_{\mathrm{xx}}}{\partial \mathrm{x}}$ & $\mathrm{S}_{\mathrm{y} 2}$ & $\frac{\partial \tau_{\mathrm{yy}}}{\partial \mathrm{y}}$ \\
\hline $\mathrm{S}_{\mathrm{x} 3}$ & $\frac{\partial \tau_{\mathrm{xy}}}{\partial \mathrm{y}}$ & $\mathrm{S}_{\mathrm{y} 3}$ & $\frac{\partial \tau_{\mathrm{xy}}}{\partial \mathrm{x}}$ \\
\hline $\mathrm{S}_{\mathrm{x} 4}$ & $-\frac{\partial}{\partial \mathrm{x}}\left(\mu \frac{\partial \mathrm{u}}{\partial \mathrm{x}}\right)$ & $\mathrm{S}_{\mathrm{y} 4}$ & $-\frac{\partial}{\partial \mathrm{y}}\left(\mu \frac{\partial \mathrm{v}}{\partial \mathrm{y}}\right)$ \\
& $-\frac{\partial}{\partial \mathrm{y}}\left(\mu \frac{\partial \mathrm{u}}{\partial \mathrm{y}}\right)$ & & $-\frac{\partial}{\partial \mathrm{x}}\left(\mu \frac{\partial \mathrm{v}}{\partial \mathrm{x}}\right)$ \\
\hline
\end{tabular}

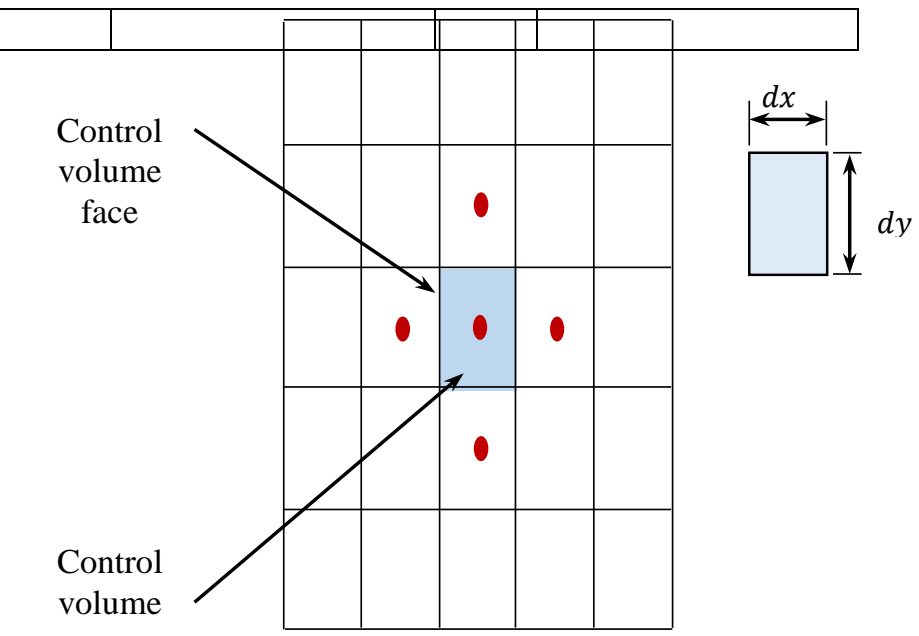

Fig.2. The shape and the distribution of the grid for the computational domain.

Before starting the integration of the momentum equation, the domain first divided into finite volume as shown in the fig. 2 .

$$
\begin{array}{r}
\int_{V_{p}}\left(\frac{\partial}{\partial x}(\rho u u)+\frac{\partial}{\partial y}(\rho v u)\right) d V=\int_{V_{p}}\left(\frac{\partial}{\partial x}\left(\mu \frac{\partial u}{\partial x}\right)+\right. \\
\left.\frac{\partial}{\partial y}\left(\mu \frac{\partial u}{\partial y}\right)\right) d V
\end{array}
$$

As the y-momentum equation integrated in the same way so it will mention the integration only for the $\mathrm{x}$ momentum.

By using divergence theorem to transfer the volume integral to a surface integral for the diffusion and the convective while the source term integrated later:

$$
\begin{gathered}
(\rho u u A)_{e}-(\rho u u A)_{w}+(\rho v u A)_{n}-(\rho v u A)_{s}= \\
\left(\mu \frac{\partial u}{\partial x} A\right)_{e}-\left(\mu \frac{\partial u}{\partial x} A\right)_{w}+\left(\mu \frac{\partial u}{\partial y} A\right)_{n}-\left(\mu \frac{\partial u}{\partial y} A\right)_{s}+ \\
S_{x} V_{p}
\end{gathered}
$$

By using the central difference for the diffusion gradient term we get:

$$
\begin{gathered}
F_{e} u_{e}-F_{w} u_{w}+F_{n} u_{n}-F_{s} u_{s}=\frac{(\mu A)_{e}}{d x}\left(u_{E}-u_{P}\right)- \\
\frac{(\mu A)_{w}}{d x}\left(u_{P}-u_{W}\right)+\frac{(\mu A)_{n}}{d y}\left(u_{\mathrm{N}}-u_{P}\right)-\frac{(\mu A)_{s}}{d y}\left(u_{P}-u_{S}\right)+ \\
S_{x} V_{p}
\end{gathered}
$$

The mass flux is denoted by $F$ with the subscribed for the specified face and the same thing for the diffusion conductance $\mathrm{D}$ with the subscribe for the specified face:

$$
\begin{gathered}
F_{e} u_{e}-F_{w} u_{w}+F_{n} u_{n}-F_{s} u_{s}=D_{e}\left(u_{E}-u_{P}\right)- \\
D_{w}\left(u_{P}-u_{\mathrm{W}}\right)+D_{n}\left(u_{N}-u_{P}\right)-D_{s}\left(u_{P}-u_{S}\right)+ \\
S_{x} V_{p}
\end{gathered}
$$


The power low scheme is used for the convective face velocities, those by employing these schemes and after arrangement; the fallowing algebraic equation is obtained:

$A_{P} \phi_{P}=A_{W} \phi_{W}+A_{E} \phi_{E}+A_{N} \phi_{N}+A_{S} \phi_{S}+b$

Where the coefficients given in the following table;

Table.2. the coefficient of the discretization algebraic

\begin{tabular}{|c|c|}
\hline $\begin{array}{c}\text { The } \\
\text { coefficient }\end{array}$ & The value \\
\hline$A_{E}$ & $D_{e} A\left(\left|P_{e}\right|\right)+\max \left[-F_{e}, 0\right]$ \\
\hline$A_{W}$ & $D_{w} A\left(\left|P_{w}\right|\right)+\max \left[F_{w}, 0\right]$ \\
\hline$A_{N}$ & $D_{n} A\left(\left|P_{n}\right|\right)+\max \left[-F_{n}, 0\right]$ \\
\hline$A_{S}$ & $D_{s} A\left(\left|P_{s}\right|\right)+\max \left[F_{s}, 0\right]$ \\
\hline$A_{\mathrm{P}}$ & $\begin{array}{c}D_{e} A\left(\left|P_{e}\right|\right)+\max \left[F_{e}, 0\right]+D_{w} A\left(\left|P_{w}\right|\right)+ \\
D_{S} A\left(\left|P_{S}\right|\right)+\max \left[-F_{S}, 0\right]-S_{P} V_{P}\end{array}$ \\
\hline$b$ & $S_{x} V_{P}$ \\
\hline
\end{tabular}

The flow rates $F_{e}, F_{w}, F_{n}$ and $F_{s}$ are calculated across the face of the control volume, the diffusion coefficient and the Peclet number are:

Table.3. the mass flux, diffusion conductance and the

\begin{tabular}{|c|c|c|}
\hline $\begin{array}{c}\text { The flow } \\
\text { rates }\end{array}$ & $\begin{array}{c}\text { The diffusion } \\
\text { coefficient }\end{array}$ & $\begin{array}{c}\text { The Peclet } \\
\text { number }\end{array}$ \\
\hline$F_{e}=(\rho u A)_{e}$ & $D_{e}=\frac{\mu A_{x}}{\delta x}$ & $P_{e}=\frac{F_{e}}{D_{e}}$ \\
\hline$F_{w}=(\rho u A)_{w}$ & $D_{w}=\frac{\mu A_{x}}{\delta x}$ & $P_{w}=\frac{F_{w}}{D_{w}}$ \\
\hline$F_{n}=(\rho v A)_{n}$ & $D_{n}=\frac{\mu A_{y}}{\delta y}$ & $P_{n}=\frac{F_{n}}{D_{n}}$ \\
\hline$F_{s}=(\rho v A)_{s}$ & $D_{s}=\frac{\mu A_{y}}{\delta y}$ & $P_{s}=\frac{F_{s}}{D_{s}}$ \\
\hline
\end{tabular}

The function $A\left(\left|P_{i}\right|\right)$ for power low scheme is [13]:

$$
A\left(\left|P_{i}\right|\right)=\max \left[0,(1-0.1|P|)^{5}\right]
$$

\subsection{Discretization of the source term}

The source term for the $\mathrm{x}$-momentum equation is spilt into four parts for clearing those:

$$
S_{x}=S_{x 1}+S_{x 2}+S_{x 3}+S_{x 4}
$$

By integration the terms individually by the using the volume integration around the cell $\mathrm{P}$

$1-\mathrm{S}_{\mathrm{x} 1}$

$$
\begin{array}{r}
\mathrm{S}_{\mathrm{x} 1}=-\frac{\partial \mathrm{P}}{\partial \mathrm{x}} \Rightarrow \int_{\mathrm{V}_{\mathrm{P}}} \mathrm{S}_{\mathrm{x} 1} \mathrm{dV}=-\int_{\mathrm{V}_{\mathrm{P}}} \frac{\partial \mathrm{P}}{\partial \mathrm{x}} \mathrm{dV}= \\
\frac{\mathrm{P}_{\mathrm{e}}-\mathrm{P}_{\mathrm{w}}}{\mathrm{dx}} \mathrm{V}_{\mathrm{P}} \quad(24)
\end{array}
$$

$2-S_{\mathrm{x} 2}$

$\mathrm{S}_{\mathrm{x} 2}=\frac{\partial \tau_{\mathrm{xx}}}{\partial \mathrm{x}} \Rightarrow \int_{\mathrm{V}_{\mathrm{P}}} \mathrm{S}_{\mathrm{x} 2} \mathrm{dV}=\frac{\tau_{\mathrm{xxe}}-\tau_{\mathrm{xxw}}}{\mathrm{dx}} \mathrm{V}_{\mathrm{P}}$

3- $\mathrm{S}_{\mathrm{x} 3}$

$S_{x 3}=\frac{\partial \tau_{x y}}{\partial y} \Rightarrow \int_{V_{P}} S_{x 3} d V=\frac{\tau_{x y n}-\tau_{x y s}}{d y} V_{P}$

4- $\mathrm{S}_{\mathrm{x} 4}$

This term is the same for the diffusion in the algebraic equation so just added it to the source term:

$S_{x 4}=-\left(D_{e}\left(u_{E}-u_{P}\right)-D_{w}\left(u_{P}-u_{W}\right)+D_{n}\left(u_{N}-u_{P}\right)\right.$

$$
\left.-D_{S}\left(u_{P}-u_{S}\right)\right)
$$

\subsection{Discretization of the constitutive equations}

The constitutive equations contain convective terms only for the primitive variable, so the divergence theorem integration will applied only for these parts. The up-wind scheme is used for the convective terms after integration is done.

Because of the similarity in the discretization of the three constitutive equations, the integration steps will mention her only for the $\mathrm{X}$-axis equation.

Normal stress $\tau_{\mathrm{xx}}$ :

$$
\begin{array}{r}
\frac{\partial}{\partial x}\left(\lambda u \tau_{x x}\right)+\frac{\partial}{\partial y}\left(\lambda v \tau_{x x}\right)=2 \mu \frac{\partial u}{\partial x}-\tau_{x x}+2 \lambda \frac{\partial u}{\partial x} \tau_{x x}+ \\
2 \lambda \frac{\partial u}{\partial y} \tau_{x y}
\end{array}
$$

By integration this equation by using divergence theorem for the convective terms:

$$
\begin{array}{r}
\left(\lambda u \tau_{x x} A\right)_{e}-\left(\lambda u \tau_{x x} A\right)_{w}+\left(\lambda v \tau_{x x} A\right)_{n}-\left(\lambda v \tau_{x x} A\right)_{s}= \\
2 \mu\left(\frac{u_{e}-u_{w}}{d x}\right) V_{P}-\tau_{x x P} V_{P}+2 \lambda\left(\frac{u_{e}-u_{w}}{d x}\right) \tau_{x x P} V_{P}+ \\
2 \lambda\left(\frac{u_{n}-u_{s}}{d y}\right) \tau_{x y P} V_{P}
\end{array}
$$

After arrangement this equation and using the up-wind scheme for the convective:

$$
A_{P} \tau_{x x P}=\sum_{F} A_{F} \tau_{x x F}+S_{1}
$$


Where the summation is done over the four neighbor of the nodal point $\mathrm{P}$. the different terms are as follows:

$$
\begin{gathered}
A_{P}=\max \left(-F_{w}, 0\right)+\max \left(F_{e}, 0\right)+\max \left(F_{n}, 0\right)+ \\
\max \left(-F_{S}, 0\right)+V_{P} \\
A_{W}=\max \left(F_{w}, 0\right), A_{E}=\max \left(-F_{e}, 0\right), A_{N}= \\
\max \left(F_{n}, 0\right), A_{S}=\max \left(-F_{s}, 0\right)
\end{gathered}
$$

$2 \lambda\left(\frac{u_{n}-u_{s}}{d y}\right) \tau_{x y P} V_{P}$

\subsection{Collected grid}

the check board pressure problem is prevented in the collected grid, by using the spatial interpolation of RhieChow [10]. Which is used to calculate the face velocity that used in the mass fluxes and the pressure correction equation.

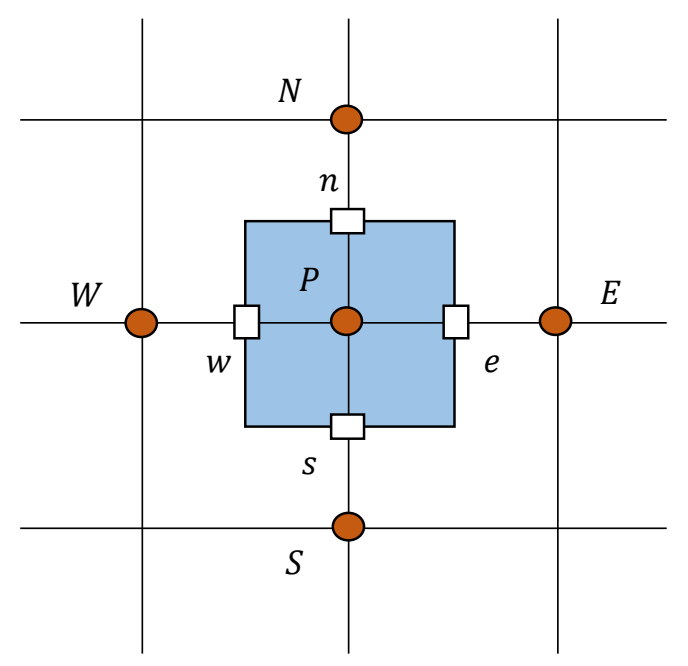

Fig.3. The face velocity position in the control volume [10].

$$
\begin{array}{r}
u_{f}=\overline{u_{f}}-\overline{B_{f}^{u}}+\overline{D_{f}^{u}} \overline{\left(\frac{\partial p}{\partial x}\right)_{f}}+\overline{B_{f}^{u}}-\overline{D_{f}^{u}}\left(\frac{\partial p}{\partial x}\right)_{f} \\
u_{f}=\overline{u_{f}}-\overline{D_{f}^{u}}\left(\left(\frac{\partial p}{\partial x}\right)_{f}-\overline{\left(\frac{\partial p}{\partial x}\right)_{f}}\right)
\end{array}
$$

This velocity used to calculate the mass flux in the momentum equation and in the pressure correction equation.

\subsection{Solution algorithm}

The solution of the algebraic equations for the momentum and the constitutive equations follows the correction procedure and the pressure correction equation which used to find the pressure field. This procedure first time used by Patankar to solve the momentum equations and to fiend the flow field variables. The algorithm for Patankar called the semi-implicit method for the pressurelinked equation (SIMPLE). Also there are other algorithms considered as a modification for the original one (SIMPLE), such as SIMPLE revised (SIMPLER) [7] and the SIMPLE consistent (SIMPLEC) algorithm.

In this simulation the SIMPLE algorithm is used. By making the required modification of the original algorithm to handle the viscoelasticity. This modification is past on the discretization of the momentum equation and the constitutive equation as it is explained earlier.

The algorithm flow is as below:

1- Assume initial value for all the variable

2- Solve the momentum equations to obtain the velocity field

3- Solve the correction equation

4- Correct the pressure and velocities

5- Solve the constitutive equations to obtain the stresses

6- Check converges, if converged stop, else go to point 1 and set the current value of variable as initial value.

Thus the solution of the allegoric equations obtain by using the TDMA(Tri-Diagonal Matrix Algorithm) which is used for the matrixes of Diagonal coefficient type or the matrixes which is all zeros just the diagonal that known to come from the discretization by the FVM .

\section{Results and discussion}

The results were obtiand by a code written in Fortran programing language in the unvirsety of Thi-Qar in Enginering college -Mechanical department by the auther. The code was adabtude to handle the viscoelastisty according to the soltion alogarthim.

The numerical simlution taken by [9] is repeated using the same differential constitutive equation. The problem is shown in fig.(1). The flow considered through sudden expansion with an expansion ratio 1:4. In this simulation the dimensionless Deborah number (De) was used to control the viscoelasticity of the fluid and it is changed in a range of values $(0,0.5,1$, and 1.5). As done by [9] $\mathrm{Re}$ Reynolds number was constant in all the simulations and it was equal to 0.1 .

The grid density used for the calculation shown in table.4for three different mesh setting.

Table.4. the mesh density used in the simulation.

\begin{tabular}{|c|c|c|c|}
\hline \multicolumn{2}{|c|}{ Mesh } & $\begin{array}{c}\text { No. of CV.in x- } \\
\text { direction }\end{array}$ & $\begin{array}{c}\text { No. of CV.in y- } \\
\text { direction }\end{array}$ \\
\hline M1 & 51 & & 80 \\
\hline M2 & 81 & 100 \\
\hline M3 & 81 & 180 \\
\hline
\end{tabular}


The first simulation is done for the Newtonian case when $\mathrm{De}=0$. In this case the circulation zone used to compare with another cases when a large Deborah numbers are used.

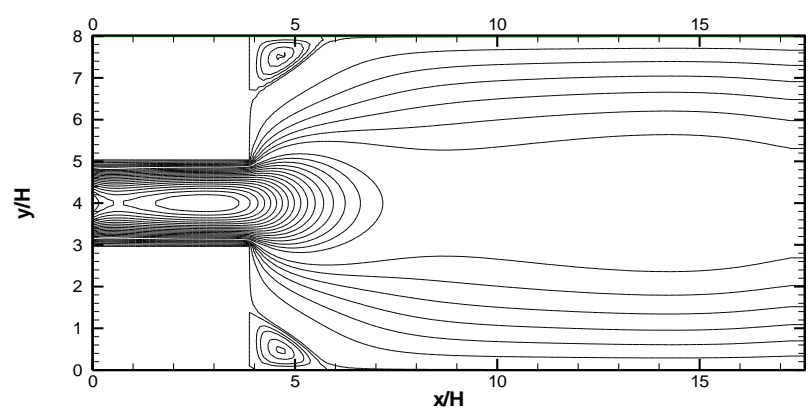

(a) Contour for the axial velocity

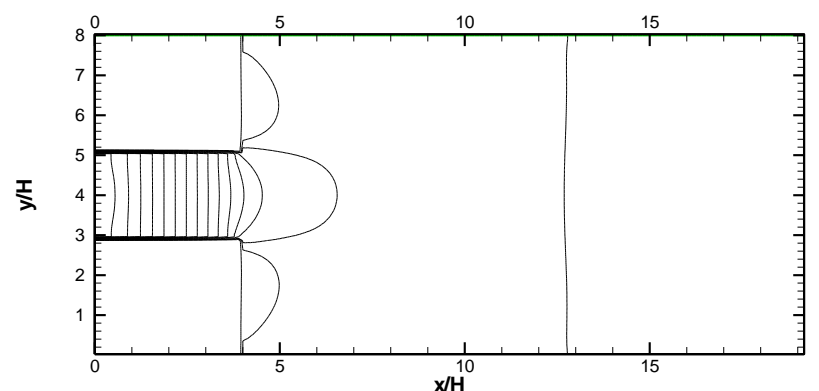

(b) Contour for pressure

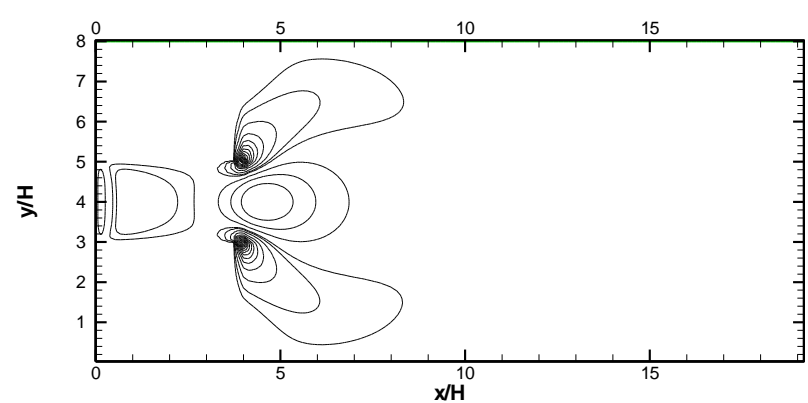

(c) Contour for the normal stress

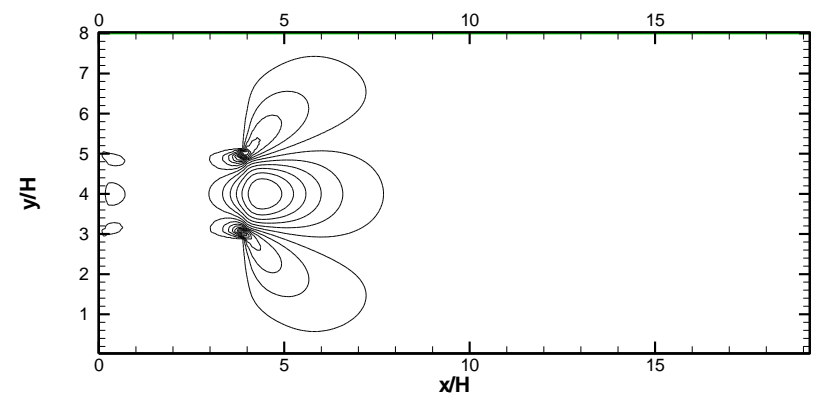

(d) Contour for the normal stress $\tau_{y y}$

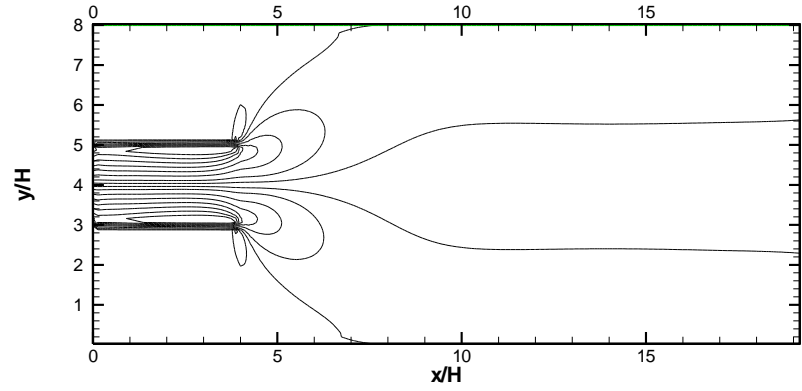

(e) Contour for the shear stress

Fig.4. The contours for the primitive variable (a) axial velocity contour (b) pressure contour (c) $\tau_{x x}$ contour (d) $\tau_{y y}$ contour (e) $\tau_{x y}$ contour, for $\mathrm{De}=0, \mathrm{Re}=0.1$.

In the figures below the results are obtained for the case of De $=0.75$.

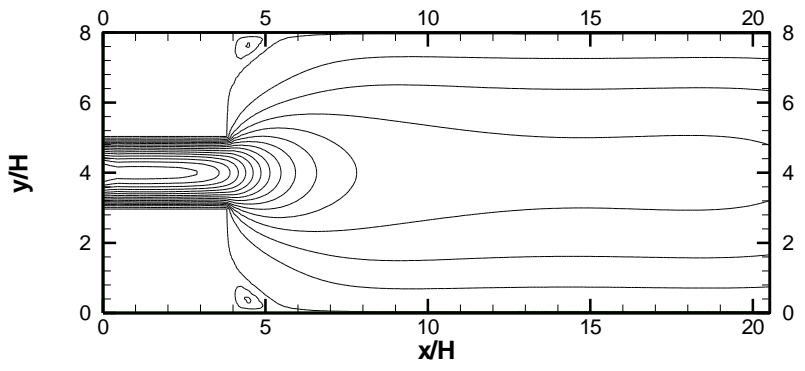

(a) Contour for the axial velocity

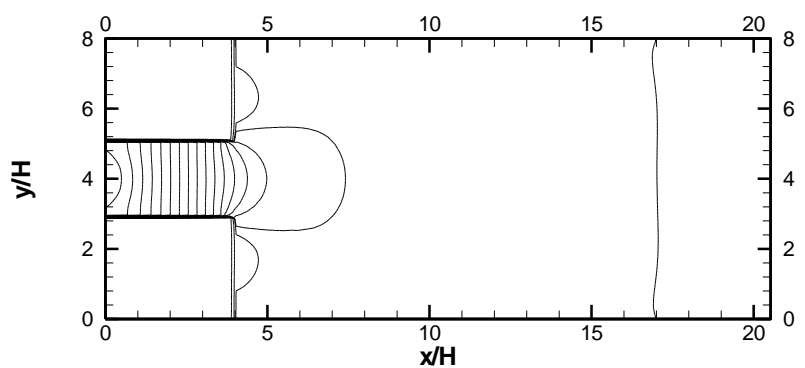

(b) Contour for pressure 


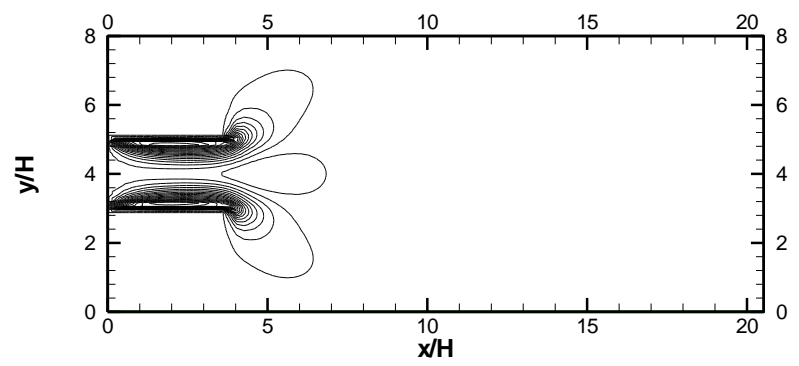

(c) Contour for the normal stress $\tau_{x x}$

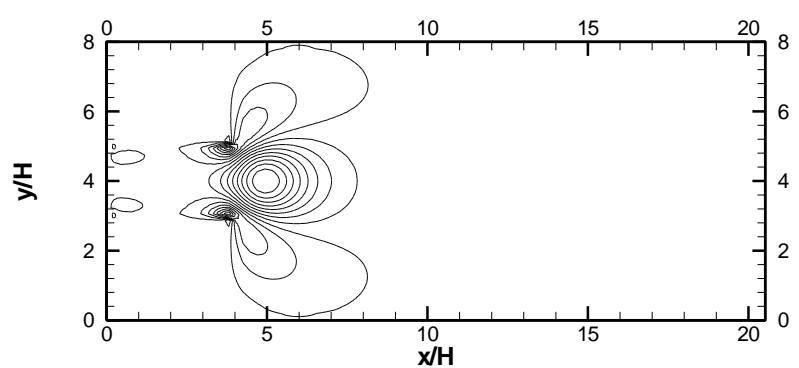

(d) Contour for the normal stress $\tau_{y y}$

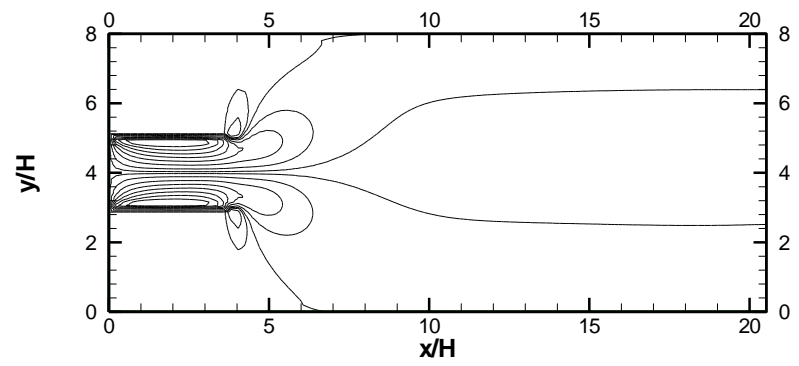

(e) Contour for the shear stress $\tau_{x y}$

Fig.5. the contours for the primitive variable (a) axial velocity contour (b) pressure contour (c) $\tau_{x x}$ contour (d) $\tau_{y y}$ contour (e) $\tau_{x y}$ contour , for $\mathrm{De}=0.75, \mathrm{Re}=0.1$.

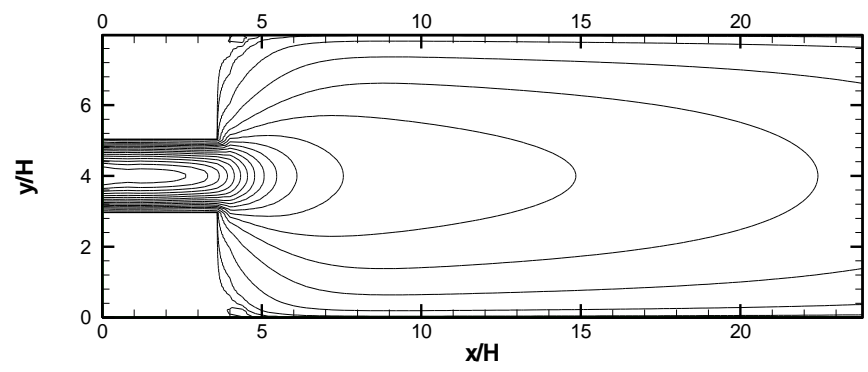

(a) Contour for the axial velocity

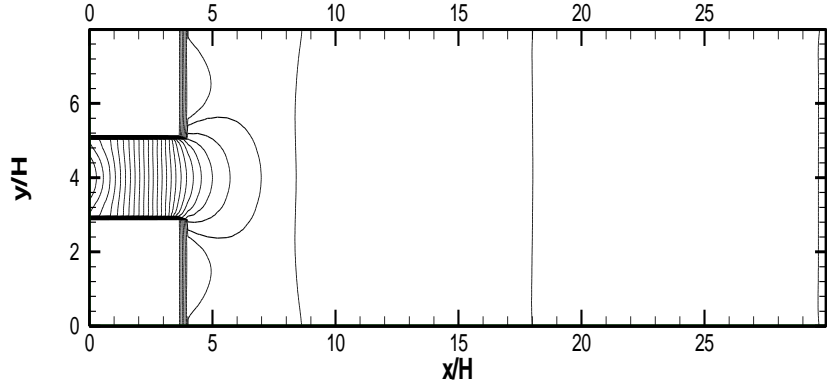

(b) Contour for pressure

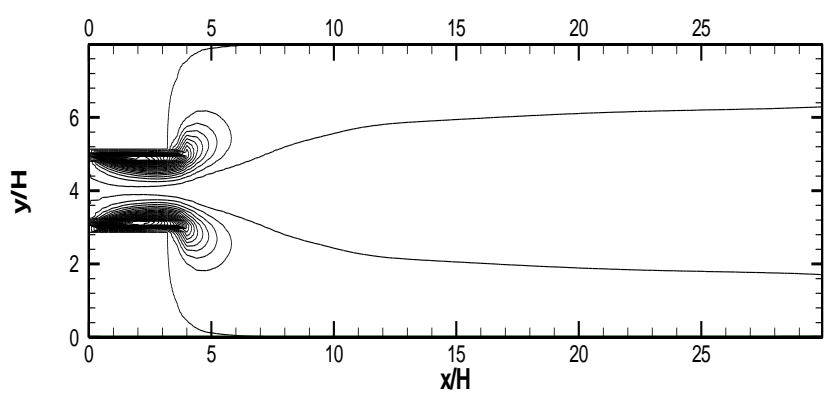

(c) Contour for normal stress $\tau_{x x}$

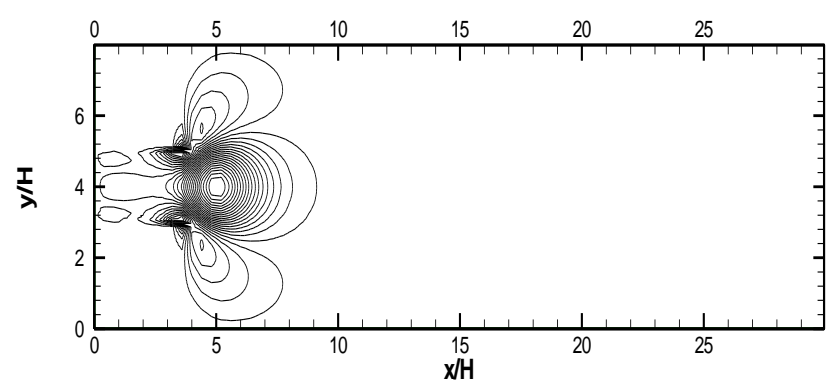

(d) Contour for normal stress $\tau_{y y}$

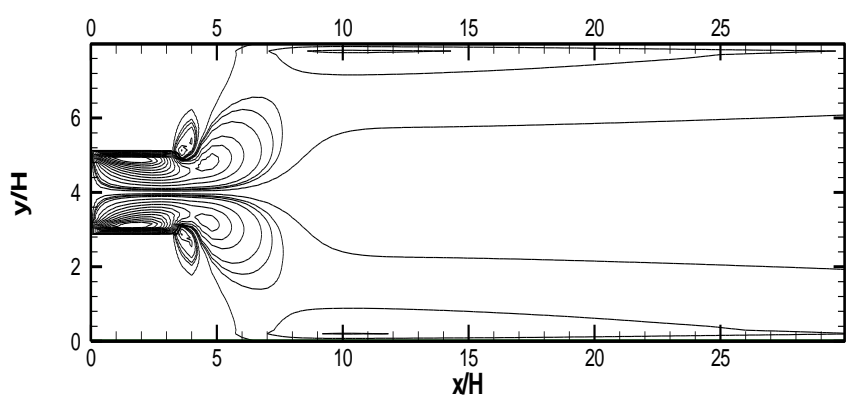

(e) Contour for shear stress $\tau_{x y}$

Fig.6. the contours for the primitive variable (a) axial velocity contour (b) pressure contour (c) $\tau_{x x}$ contour (d) $\tau_{y y}$ contour (e) $\tau_{x y}$ contour , for $\mathrm{De}=1.5, \mathrm{Re}=0.1$.

It is observed that as Deborah number increased the computational domain length also need to increase. Those 
the first simulation is for the Newtonian case when $\mathrm{De}=0$ the computational domain was have a length of $20 \mathrm{H}$ and this length was good for the stability of the numerical method but. If Deborah numbers became higher than 0.75 the length of $20 \mathrm{H}$ was not good for the stability of the numerical method and in the case of $\mathrm{De}=1.5$ the length of $30 \mathrm{H}$ was good for stability of the solution so the result was obtain for this length .

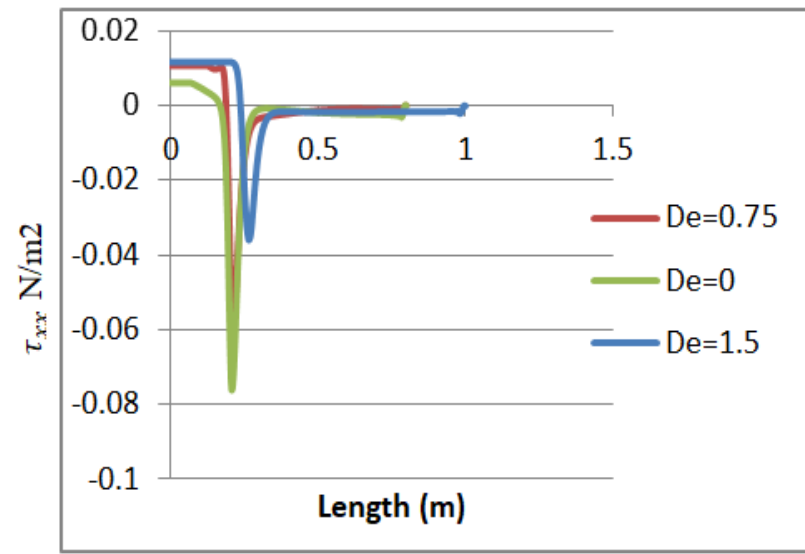

Fig.7. $\tau_{x x}$ along the center line for different De numbers, for $\mathrm{Re}=0.1$.

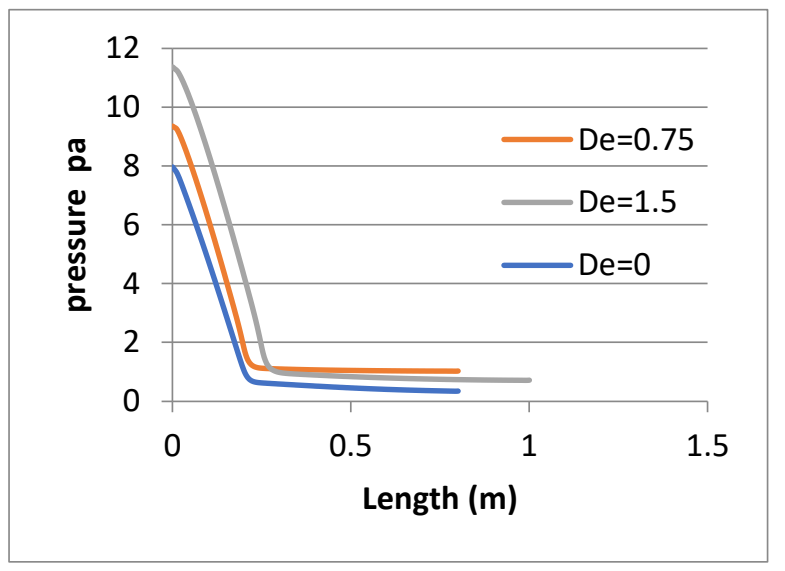

Fig.8. The pressure along the center line for different De numbers, for $\mathrm{Re}=0.1$.

Fig.5. shows the contours for the (a) axial velocity contour (b) pressure contour (c) $\tau_{x x}$ contour (d) $\tau_{y y}$ contour (e) $\tau_{x y}$ contour, at $\mathrm{De}=0.75, \mathrm{Re}=0.1$. It is clear from the figure that the recirculation zones are smaller than that at Fig.4 in the Newtonian case. Also the difference can be seen for the pressure contour and the stresses cotours .

In Fig.6 it can be seen that the effect of Deborah number on the circulation zones is to reduce their size.
They are nearly eliminated from the contour, this happened because the increasing of the elasticity levels (when De number increase) making the fluid moves adjacent to the wall of the pipe. In the Newtonian case when entering a channel with a larger height, the stresses will relaxed along the streamlines. But For the viscoelastic fluids this will causes expansion in the transverse flow direction.

In the same way from fig. 8 and fig. 7 it is observed that the pressure drop and the pressure required to push the fluid inside the channel both were increased and this happened because of the high level of elasticity making the fluid giving more resistance for deformation and movement.

Fig.7 shows the normal stress $\tau_{\mathrm{xx}}$ at the center line of the channel. It shows that this stress was increased when De number increase, where at $\mathrm{De}=0$ having small value at the expansion or at the entrance to the channel while at $\mathrm{De}=1.5$ the difference clearly appear. The same reason here because of the high elasticity levels making the fluid give more resistance to deform not like the Newtonian fluid where the deformation is easily happened.

\section{Concluding remarks}

Using FVM for the numerical simulation in general offer a simple implementation and has the power and the ability to deal with complex partial differential equations .In this work that done for the UCM viscoelastic fluid flow in a 1:4 expansion duct were a stabile solution is obtained up to Deborah number equal to 1.5 and the flow was considered to be creeping flow at Reynolds number 0.1 . The results shows that the increasing of the viscoelasticity levels leads to reducing the recirculation zones that generated at the corner of the expansion also the pressure drop was increased when the elasticity level increased.

\section{References}

[1] Beris, Armstrong And Brown. 1987. Spectral Finite Element Calculations Of The Flow Of A Maxwell Fluid Between Eccentric Rotating Cylinders. 1987.

[2] Çengel, Yunus. 2014. Fluid Mechanics Fundamentals And Applications. 1221 Avenue Of : Mcgraw-Hill, 2014.

[3] Chang, Patten And Finlayson. 1979. Collocation And Galerkin Finite Element Methods For Viscoelastic Fluid Flow. S.L. : Comput. Fluids, 1979.

[4] Chun-Quan, Fu. 2009. Finite Volume Method For Simulation Of Viscoelastic Fluid Flow. Daqing 163001, China : Elsevier, 2009. 
[5] Gasti, T.B. 1975. Steady Flow Of A Non-Newtonian Fluid Through A Contraction. S.L. : J. Comput., 1975.

[6] Kerim Yapici, Bulent Karasozen. 2010. Finite Volume Simulation Of Viscoelastic Laminar Flow In A Lid-Driven. Ankara, Turkey: Middle East Technical University, 2010.

[7] Malalasekera, H. K. Versteeg And W. 2007. An Introduction To Computational Fluid Dynamics. London : Pearson, 2007.

[8] Michel O. Deville, Thomas B. Gatski. 2010. Mathematical Modeling For Complex Fluids And Flow. Lausanne, Switzerland : Springer, 2010.

[9] Missirlis, Assimacopoulosa And Mitsoulis. 1998. A Finite Volume Approach In The Simulation Of Viscoelastic Expansion Flows. Athens 157 -80, Greece: Elsevier, 1998.

[10] Moukalled, Mangani And Darwish. 2015. The Finite Volume Method In Computational Fluid Dynamics. Beirut : Openfoam, 2015.

[11] Oliveira A, Pinho And Pinto. 1998. Numerical Simulation Of Non-Linear Elastic Flows With A General Collocated Finite-Volume Method. 6200 Co6ilha , Portugal : Elsevier, 1998.

[12] Oliveira, Paulo And. 2003. Asymmetric Flows Of Viscoelastic Fluids In Symmetric Planar Expansion Geometries. 6201-001 Covilhã, Portugal : Elsevier, 2003.

[13] Sahus.V.Patankar. 1980. Numerical Heat Transfear And Fluid Flow. Newyork : S.N., 1980.

[14] Walters, Perera And. 1977. Long-Range Memory Effects In Flows Involving Abrupt Changes In Geometry. Part I.Flows Associated With L-Shaped And T-Shaped Geometries. 1977.

[16] Whiteman, M.S. Dar-Wish And J.R. 1992. Numerical Modelling Of Viscoelastic Liquids Using A Finite-Volume Method. Uxbridge Ub8 3ph, Uk: Department Of Mathematics And Statistics, 1992.

[17] Paulo J. Oliveira. Method For Time-Dependent Simulations of Viscoelastic Flows:Vortex Shedding Behind Cylinder. Covilhã, Portugal, Elsevier, 2001. [18] Alves, Pinho and Oliveira. The Flow Of Viscoelastic Fluids Past A Cylinder: Finite-Volume HighResolution Methods. Covilhã, Portugal, Elsevier, 2001
Numerical Simulation Of Viscoelastic Turbulent Channel Flow Exhibiting Drag Reduction: Effect Of The Variation Of Rheological Parameters. Newark, Elsevier, 1998. [21] Azaiez, Gu6nette and Ait-Kadi. Numerical Simulation Of Viscoelastic Flows Through A Planarcontraction. Quebec, Canada, Elsevier, 1995. [22] Dar-Wish And Whiteman. Numerical Modelling Of Viscoelastic Liquids Using A Finite-Volume Method. Brunei University,, Elsevier, 199.

[23] Poole, Alves B and Oliveira. Plane Sudden Expansion Flows of Viscoelastic Liquids . Braga, Portugal, Elsevier, 2007.

[24] Keunings And Crochet. Numerical Simulation Of The Flow Of A Fluid Through An Abrupt Contraction.

Amsterdam, Elsevier, 1984.

[25] Khezzar, Filali and Alshehhi. Flow And Heat Transfer Of Fene-P Fluids In Ducts Of Various Shapes:Effect Of Newtonian Solvent Contribution. United Arab Emirates, Elsevier, 2014.

[20] Costas , Dimitropoulosa and Sureshkumar. Direct 